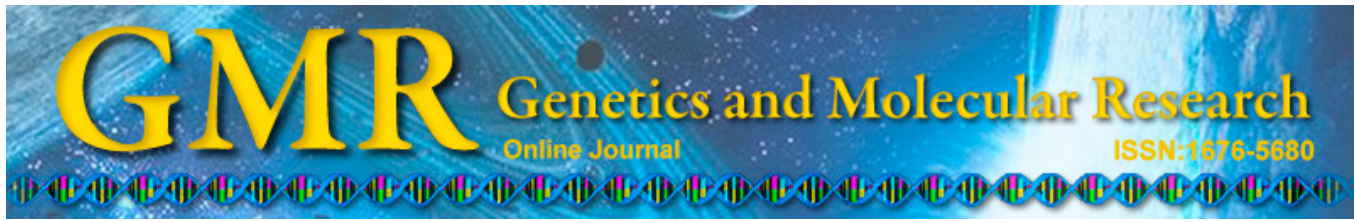

\title{
Relationship between UV-irradiated HaCaT cell cytokines and Th1/Th2 imbalance
}

\author{
H.Y. Li ${ }^{1,2}$, F.R. Zhang ${ }^{1,3}$ and D.Q. Deng ${ }^{4}$ \\ ${ }^{1}$ Shandong Provincial Hospital for Skin Diseases, Shandong University, \\ Jinan, Shandong, China \\ ${ }^{2}$ Department of Dermatology, \\ The Affiliated Hospital of Binzhou Medical University, Binzhou, \\ Shandong, China \\ ${ }^{3}$ Shandong Provincial Institute of Dermatology and Venereology, \\ Jinan, Shandong, China \\ ${ }^{4}$ Department of Dermatology, \\ The Second Affiliated Hospital of Kunming Medical University, \\ Kunming, Yunnan, China \\ Corresponding author: D.Q. Deng \\ E-mail: lihaiying_1@163.com
}

Genet. Mol. Res. 14 (3): 7976-7985 (2015)

Received September 30, 2014

Accepted April 28, 2015

Published July 17, 2015

DOI http://dx.doi.org/10.4238/2015.July.17.5

ABSTRACT. We have previously found that an imbalance of Tc1/Tc2
T cell subtypes in vivo impacts the development of photodermatitis. The
aim of this study was to investigate the relationship between cytokines
derived from keratinocytes exposure to UV and the imbalance of Th
subgroups. We used different doses of UVA and UVB to irradiate
HaCaT cells. Twelve hours after irradiation, the expression of IL-
10R, IL-4R, IL-12R, and IFN- $\gamma \mathrm{R}$ proteins was observed using the S-P
method, and the percentage of positive cells calculated. Protein levels of
the respective ligands in the supernatant was measured by ELISA. Our
results showed low levels of expression of the interrogated proteins in
unirradiated HaCaT cells, and little or no expression could be detected
in the supernatant. Little or no expression was also observed for IL-12R 
and IFN- $\gamma \mathrm{R} 12 \mathrm{~h}$ after UVA or UVB irradiation. However, the expression of IL-10R and IL-10 was upregulated $12 \mathrm{~h}$ following UVB irradiation, as well as following lower dose UVA irradiation. In contrast, higher dose UVA decreased the expression of IL-10R and IL-10. The expression of IL-4R was increased following high doses of UVA and UVB irradiation, whereas no expression was observed after lower dose UV exposure. There was no change in IL-4 secretion into the supernatant. Our results demonstrate that the effects of UV exposure on keratinocyte-derived cytokines are different according to the doses of irradiation and the types of cytokines, and suggest that keratinocyte-derived cytokines after UV exposure might cause an imbalance of Th1/Th2.

Key words: Irradiation; HaCaT cells; Cytokines; Th cells

\section{INTRODUCTION}

The immune system of the skin is constituted of various immunocytes; among these, the keratinocyte is of particular importance, as it can secrete various cytokines, thus participating in immunoregulation. Keratinocytes have a primary role in the amplification of skin inflammation during immune-mediated skin diseases.

Over the last 10 years, a large number of studies have focused on the effects of ultraviolet (UV) radiation on keratinocyte-derived cytokines and have reported that the relationship between keratinocyte-derived cytokines and the imbalance of T helper cell subtype (Th1/Th2) ratio had some impact on the occurrence and development of certain skin diseases (Mozzanica et al., 1995; Teraki et al., 2000). Our previous research in vivo indicated that the infiltrating monocytes isolated from patient epidermis and dermis were primarily composed of CD8+ T cells expressing IFN- $\gamma$ and IL-12 receptors. At the same time, the IFN- $\gamma$ and IL-12 levels detected in patient serum were higher compared to those of normal controls, which indicated that the imbalance of the $\mathrm{T}$ cell subtype (Tc1/Tc2) ratio had some impact on the development of photo-dermatitis. Based on our previous study, we used UVA and UVB to irradiate human immortal epidermis (HaCaT) cells, and then detected the expression of IL-10R, IL-4R, IL-12R, and IFN- $\gamma \mathrm{R}$ in the cells and the secretion of IL-10, IL-4, IL-12, and IFN- $\gamma$ in the serum $12 \mathrm{~h}$ after irradiation to investigate the impact of UV irradiation on keratinocyte-derived cytokines and the relevance of the cytokines to the imbalance of $T$ cell subgroups. These studies will provide theoretical and experimental evidence for further research into the pathogenesis of photo-dermatitis.

\section{MATERIAL AND METHODS}

\section{Radiation sources}

In this study, we utilized ultraviolet phototherapy apparatus models SS-01 (spectrum 320-400 nm, peak $370 \mathrm{~nm}$ ) and SS-01B (spectrum $312 \pm 10 \mathrm{~nm}$ ) (Sigmar High-tech Limited, Shanghai, China). By fixing the irradiation distance, the irradiation intensity was also fixed. We could obtain different irradiation doses according to different irradiation times. In this study, we irradiated cells with UVA for 62 and $128 \mathrm{~s}$, and the corresponding irradiation doses 
were 2.00 and $4.00 \mathrm{~J} / \mathrm{cm}^{2}$, respectively. Similarly, the irradiation times for UVB were 10, 20, and $40 \mathrm{~s}$, and the corresponding irradiation doses were $30.00,60.00$, and $120.00 \mathrm{~mJ} / \mathrm{cm}^{2}$. Before irradiation, the cell culture medium (Dulbecco's modified Eagle's medium, DMEM) was replaced with phosphate buffered saline (PBS).

\section{Cell lines, antibodies, and reagents}

The HaCaT cell line was purchased from China Type Culture Conserve Center at Wuhan College, Wuhan, China. For immunohistochemical staining, rabbit polyclonal antihuman IL-10R (SC-984), IL-12R 1 1 (SC-658), IL-4R (SC-684), and IFN- $\gamma$ R (SC-700) antibodies were used (Santa Cruz Biotechnology, Santa Cruz, CA, USA). ELISA kits for IL-10, IL-12, IL-4, and IFN- $\gamma$ were also used (JingMei Biotechnology Company, Beijing, China). Cell culture medium, supplements, and fetal bovine serum (FBS) were obtained from GIBCO (Gaithersburg, MD, USA)

\section{In vitro $\mathrm{UV}$ irradiation of $\mathrm{HaCaT}$ cells}

The HaCaT cell line was grown in DMEM supplemented with 10\% FBS. When the desired cell number was reached, the cells were dissociated and the suspensions were added to $40-\mathrm{mm}$ culture dishes at $2.5 \times 10^{5} / \mathrm{mL}$ in $2 \mathrm{~mL} \mathrm{DMEM}$ with $10 \% \mathrm{FBS}$ and cultured at $37^{\circ} \mathrm{C}$, $5 \% \mathrm{CO}_{2}$. When $70-75 \%$ confluence was achieved, the medium was removed and the cells were rinsed three times with PBS and overlain with $0.1 \mathrm{~mL}$ PBS. The HaCaT cells were irradiated with different doses of UVA and UVB. After irradiation, the cells were cultured in DMEM with $10 \% \mathrm{FBS}$ at $37^{\circ} \mathrm{C}$ with $5 \% \mathrm{CO}_{2}$. At $12 \mathrm{~h}$ following irradiation, the supernatant fluid was removed and centrifuged for $10 \mathrm{~min}$ at $4000 \mathrm{rpm}$, and stored at $-70^{\circ} \mathrm{C}$ for later use. The cells were dissociated and spotted onto slides. The slides were dehydrated in $95 \%$ alcohol, rinsed with PBS, and air dried at room temperature. The cells on the slides were fixed with $4{ }^{\circ} \mathrm{C}$ acetone for $10 \mathrm{~min}$ for later use.

\section{Immunohistochemistry}

In preparation for immunohistochemical analysis, fixed sample slides were rinsed with PBS and dried at room temperature. They were treated with endogenous peroxidase inhibitor for 10 min to quench endogenous peroxidase activity, and rinsed three times with PBS for 3 min each. Slides were subsequently treated with normal animal serum for 10 min, following which the serum was dispelled without additional rinsing. The slides were incubated with anti-IL-10R, anti-IL-12R, anti-IL-4R, and anti-IFN- $\gamma \mathrm{R}$ separately for $2 \mathrm{~h}$ at room temperature, and then rinsed with three times with PBS for 3 min each. Biotin-labeled secondaryAbs were applied for $10 \mathrm{~min}$, and then the slides were subjected to another PBS rinse series. Samples were treated with streptavidin-peroxidase at room temperature for 10 min (Maixin Biotechnology Development Company in Fuzhou), followed by PBS rinses as before. Staining was performed with $\mathrm{DAB}$ for $10 \mathrm{~min}$. Slides were re-stained with hematoxylin for $2 \mathrm{~min}$, differentiated with hydrochloric acid-alcohol for $2 \mathrm{~s}$, and rinsed with water. The slides were covered with neutral gum for preservation and visualization. For the negative controls, primary Abs were omitted and replaced with PBS. 


\section{ELISA}

UV induced IL-10, IL-12, IL-4, and IFN- $\gamma$ were assayed using ELISA. According to the manufacturer protocol (JingMei Biotechnology Company). The plates were analyzed in an ELISA microplate reader (BIO-RAD model 550, Berkeley, CA, USA). DMEM was used as the zero standard. Cultures were conducted in triplicate for each condition.

\section{Ascertainment of results}

For immunohistochemistry, brown-yellow staining was taken as positive. To ascertain the percentage of positive cells, we counted three high-power fields per slide and conducted the same process for each condition. For ELISA, results were given as $\mathrm{pg} / \mathrm{mL}$ directly from the ELISA microplate reader.

\section{Statistical analysis}

Comparisons were done by one-way ANOVA if the values were normally distributed and with homogenous variance. If not, the Kruskal-Wallis rank test was used. P value $<0.05$ was considered to reflect statistical significance, and then the Mann-Whitney rank test was used to compare the difference between the experimental and control groups.

\section{RESULTS}

\section{Morphological changes}

The cell connections were loosened after UV irradiation. Refraction was weakened. Some lethality was observed. Changes in groups receiving higher doses of UV (UVA $4.00 \mathrm{~J} / \mathrm{cm}^{2}$, UVB $120.00 \mathrm{~mJ} / \mathrm{cm}^{2}$ ) were more distinct (Figures 1 and 2).

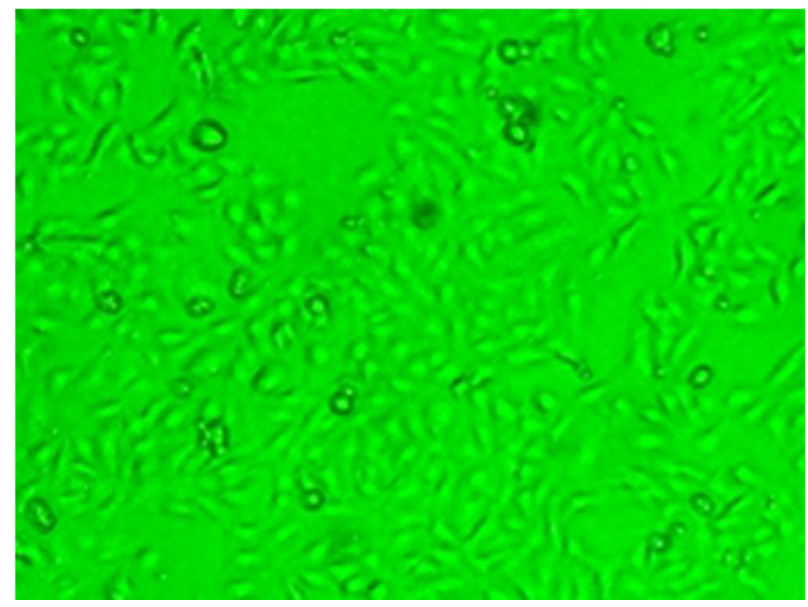

Figure 1. Twelve hours after UVA $4.00 \mathrm{~J} / \mathrm{cm}^{2}$ irradiation. 


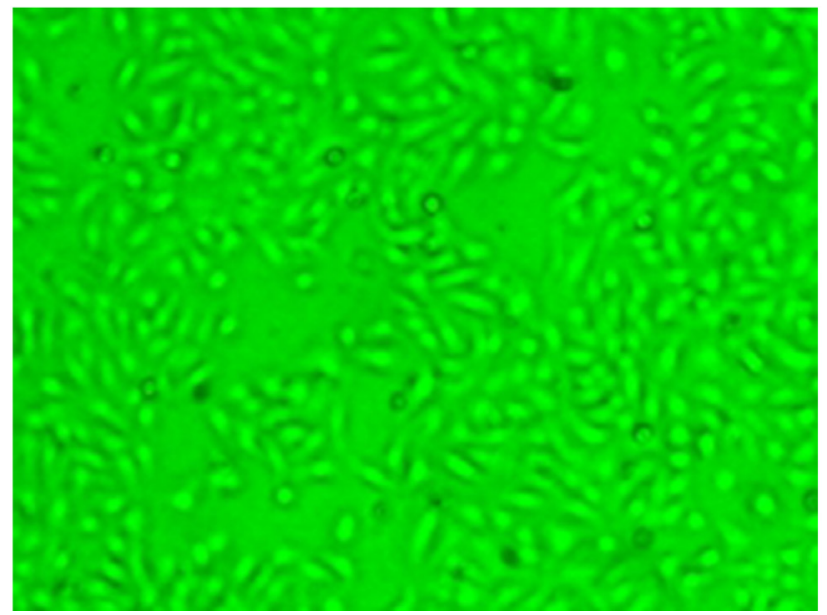

Figure 2. Twelve hours after UVB $120.00 \mathrm{~mJ} / \mathrm{cm}^{2}$ irradiation.

\section{Immunohistochemistry}

Twelve hours after UV irradiation, most $\mathrm{HaCaT}$ cell membranes and/or plasma stained positive by immunohistochemical assessment. The expression of IL-12R and IFN- $\gamma$ R was feebly positive (Figures 3A-E and 4A-E). The percentages of positive cells for these markers are listed in Table 1 . HaCaT cell membranes and/or plasma were stained slightly brown-yellow in the non-irradiated group, and the expression of IL-4R was weakly positive. At $12 \mathrm{~h}$ after UV irradiation, the expression of IL-4R was intensified, especially in the groups receiving higher doses of UV irradiation (UVA $4.00 \mathrm{~J} / \mathrm{cm}^{2}, \mathrm{UVB} 120.00 \mathrm{~mJ} / \mathrm{cm}^{2}$ (Figure 5A-E). The expression of IL-10R was strongly positive after UVB and $2.00 \mathrm{~J} / \mathrm{cm}^{2} \mathrm{UVA}$ irradiation, and weakly positive expression was seen in the $4.00 \mathrm{~J} / \mathrm{cm}^{2}$ UVA irradiation group (Figure 6A-E). The percentages of positive cells for these markers are listed in Table 2 .

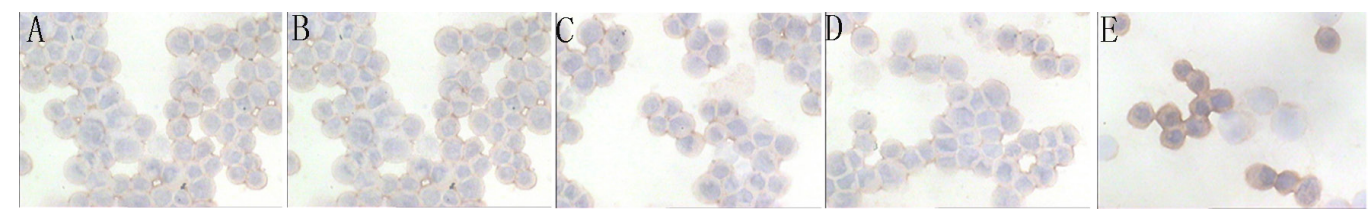

Figure 3. IFN- $\gamma$ R (S-P method x 400 times). Representative images are shown for each group: A. UVA $2.00 \mathrm{~J} / \mathrm{cm}^{2}$; B. UVA $4.00 \mathrm{~J} / \mathrm{cm}^{2}$; C. UVB $30.00 \mathrm{~mJ} / \mathrm{cm}^{2}$; D. UVB $60.00 \mathrm{~mJ} / \mathrm{cm}^{2}$; and E. UVB $120.00 \mathrm{~mJ} / \mathrm{cm}^{2}$.

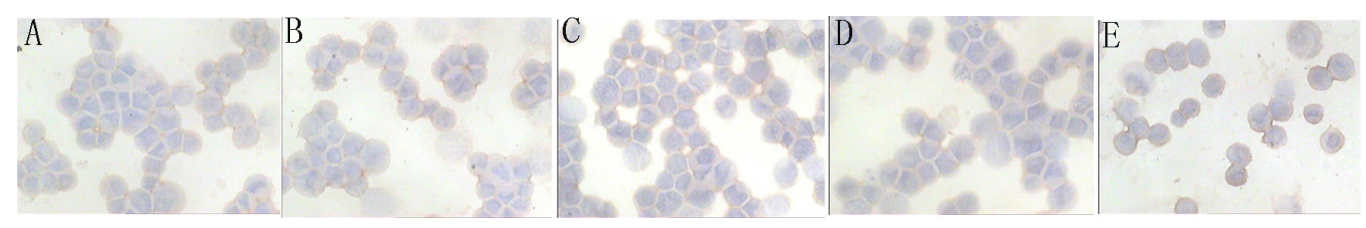

Figure 4. IL-12R (S-P method x 400 times). Representative images are shown for each group: A. UVA $2.00 \mathrm{~J} / \mathrm{cm}^{2}$; B. UVA $4.00 \mathrm{~J} / \mathrm{cm}^{2}$; C. UVB $30.00 \mathrm{~mJ} / \mathrm{cm}^{2}$; D. UVB $60.00 \mathrm{~mJ} / \mathrm{cm}^{2}$; and E. UVB $120.00 \mathrm{~mJ} / \mathrm{cm}^{2}$. 
Table 1. Percentages of IFN- $\gamma \mathrm{R}$ and IL-12R positive HaCaT cells $12 \mathrm{~h}$ after UV irradiation.

\begin{tabular}{|c|c|c|c|c|}
\hline \multirow[t]{2}{*}{ Irradiation group } & \multicolumn{2}{|c|}{ IFN- $\gamma \mathrm{R}$} & \multicolumn{2}{|c|}{ IL-12R } \\
\hline & Means \pm SD & $P$ value & Means \pm SD & $P$ value \\
\hline UVA $2 \mathrm{~J} / \mathrm{cm}^{2}$ & $94.898 \pm 1.712$ & 0.175 & $91.990 \pm 2.504$ & 0.058 \\
\hline UVA $4 \mathrm{~J} / \mathrm{cm}^{2}$ & $94.674 \pm 1.451$ & 0.230 & $90.063 \pm 3.542$ & 0.425 \\
\hline UVB $30 \mathrm{~mJ} / \mathrm{cm}^{2}$ & $91.566 \pm 2.297$ & 0.292 & $86.173 \pm 2.561$ & 0.133 \\
\hline UVB $60 \mathrm{~mJ} / \mathrm{cm}^{2}$ & $92.158 \pm 2.947$ & 0.531 & $91.077 \pm 4.289$ & 0.165 \\
\hline UVB $120 \mathrm{~mJ} / \mathrm{cm}^{2}$ & $94.884 \pm 1.946$ & 0.178 & $91.706 \pm 0.662$ & 0.082 \\
\hline $\mathrm{N}$ group & $93.016 \pm 3.196$ & & $88.728 \pm 2.232$ & \\
\hline
\end{tabular}

$\mathrm{P}>0.05$ : no significant difference between trial and control groups.

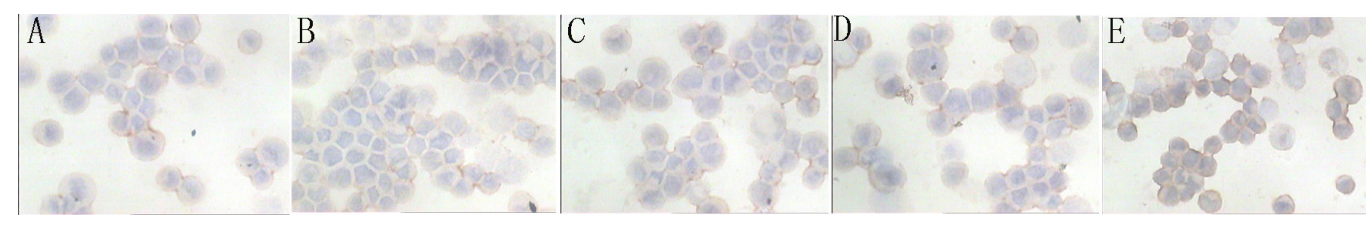

Figure 5. IL-4R (S-P immunohistochemical method $\mathrm{x} 400$ times). Representative images are shown for each group: A. UVA $2.00 \mathrm{~J} / \mathrm{cm}^{2} ;$ B. UVA $4.00 \mathrm{~J} / \mathrm{cm}^{2} ;$ C. UVB $30.00 \mathrm{~mJ} / \mathrm{cm}^{2} ;$ D. UVB $60.00 \mathrm{~mJ} / \mathrm{cm}^{2}$; and E. UVB $120.00 \mathrm{~mJ} / \mathrm{cm}^{2}$.

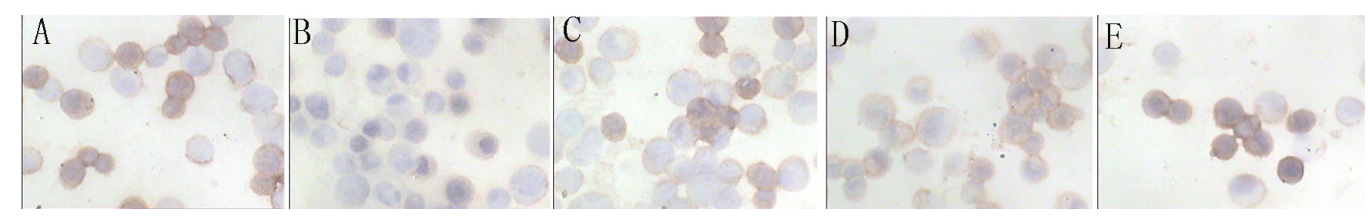

Figure 6. IL-10R (S-P immunohistochemical method $\mathrm{x} 400$ times). Representative images are shown for each group: A. UVA $2.00 \mathrm{~J} / \mathrm{cm}^{2}$; B. UVA $4.00 \mathrm{~J} / \mathrm{cm}^{2}$; C. UVB $30.00 \mathrm{~mJ} / \mathrm{cm}^{2} ;$ D. UVB $60.00 \mathrm{~mJ} / \mathrm{cm}^{2}$; and E. UVB $120.00 \mathrm{~mJ} / \mathrm{cm}^{2}$.

Table 2. Percentages of IL-4R and IL-10R positive cells in HaCaT cells $12 \mathrm{~h}$ after UV irradiation.

\begin{tabular}{|c|c|c|c|c|}
\hline \multirow[t]{2}{*}{ Irradiation group } & \multicolumn{2}{|c|}{ IL-4R } & \multicolumn{2}{|c|}{ IL-10R*** } \\
\hline & Means \pm SD & $P$ value & Means \pm SD & $P$ value \\
\hline UVA $2 \mathrm{~J} / \mathrm{cm}^{2}$ & $87.504 \pm 2.561$ & 0.088 & $96.354 \pm 1.862$ & 0.002 \\
\hline UVA $4 \mathrm{~J} / \mathrm{cm}^{2}$ & $90.053 \pm 6.073 *$ & $0.020^{*}$ & $66.318 \pm 10.976$ & 0.002 \\
\hline UVB $30 \mathrm{~mJ} / \mathrm{cm}^{2}$ & $87.623 \pm 5.852$ & 0.082 & $92.659 \pm 1.736$ & 0.026 \\
\hline UVB $60 \mathrm{~mJ} / \mathrm{cm}^{2}$ & $86.692 \pm 2.915$ & 0.130 & $95.670 \pm 2.215$ & 0.004 \\
\hline UVB $120 \mathrm{~mJ} / \mathrm{cm}^{2}$ & $90.075 \pm 4.102 * *$ & $0.020^{* *}$ & $97.601 \pm 2.109$ & 0.002 \\
\hline $\mathrm{N}$ group & $80.950 \pm 12.035$ & & $84.112 \pm 7.014$ & \\
\hline
\end{tabular}

$* \mathrm{P}=0.02<0.05$, significant difference between UVA $4 \mathrm{~J} / \mathrm{cm}^{2}$ and non-irradiated groups. ${ }^{* *} \mathrm{P}=0.02<0.05$, significant difference between UVB $120 \mathrm{~mJ} / \mathrm{cm}^{2}$ and non-irradiated groups. ${ }^{* * *}$ Using the rank test, significant difference between the trial and control groups.

\section{ELISA}

There was no or little secreted IL-12 and IFN- $\gamma$ in the supernatant at $12 \mathrm{~h}$ after UV irradiation. The Kruskal-Wallis rank test was used to compare the secretion of IL-12 and IFN- $\gamma$. $\chi^{2}$ values were 5.606 and 4.697, respectively, and $P$ values were 0.346 and 0.454 , respectively. There was no significant difference in the total distribution among the trial and control groups. Levels of secreted IL-4 and IL-10 at $12 \mathrm{~h}$ after UV irradiation are shown in Table 3. 


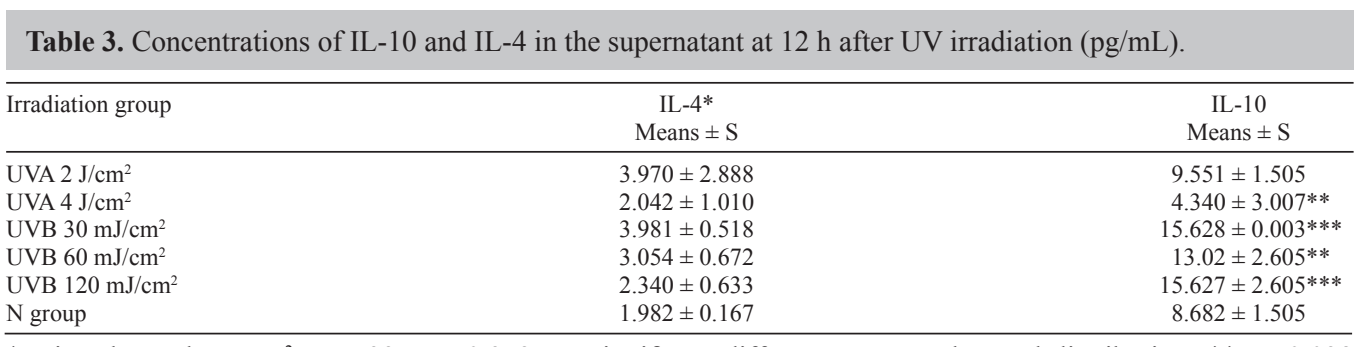

*Using the rank test, $\chi^{2}=5.532, \mathrm{P}=0.352$; no significant difference among the total distribution. $* * \mathrm{P}=0.028$ $<0.05$; significant difference between UVA4 $\mathrm{J} / \mathrm{cm}^{2}$ and non-irradiated groups. ${ }^{* * *} \mathrm{P}=0.002<0.05$; significant difference between UVB30 $\mathrm{mJ} / \mathrm{cm}^{2}$ and non-irradiated groups.

\section{DISCUSSION}

Photo-dermatitis is a spectrum disease caused by exposure to sunlight or other rays on the skin. Polymorphous light eruption (PLE) and chronic actinic dermatitis are the most common manifestations and are characterized by an intense infiltrate of monocytes in the dermis. The pathogenesis of these diseases is not clear. Recently, researchers have posited the hypothesis that delayed type hypersensitivity (DTH) is caused by skin induction exposure to sunlight. The majority of the human epidermis, approximately $80 \%$, is composed of keratinocytes. These can secrete various cytokines such as IL-1, IL-6, IL-8, IL-10, and TNF- $\gamma$ among others, and thus participate in immunoregulation. Several studies have reported that keratinocyte-derived cytokines following exposure to UV lead to pathophysiological changes by affecting the balance of Th1/Th2; however, most studies have focused on the immunosuppression aspect of cytokine production (Schmitt et al., 2000; Aubin, 2003). The study regarding diseases related to the imbalance of Th1/Th2 focused primarily on atopic dermatitis (Th1 type skin disease) and psoriasis (Th2 type skin disease). The relationship between keratinocyte-derived cytokines following exposure to UV and the Th1/Th2 imbalance has not been reported. Comparatively, our previous studies have suggested that the development of photo-dermatitis might be related to the excess expression of Tc1 and the suppression of Tc2 expression (Chen et al., 2005).

We used UVA and UVB to irradiate HaCaT cells to investigate the effects of UV irradiation on keratinocyte-derived cytokines and the relationship between cytokines and the imbalance of Th1/Th2, and to provide experimental and theoretical evidence for future studies. The HaCaT cell line consists of transfected human epidermal cells, and has the differentiation characteristics of basal layer cells. Mee et al. (2000) suggested that the effect of culture condition should be considered when discussing the cell biology in vitro. Our experiments indicated that if $\mathrm{HaCaT}$ cells were seeded at $2 \times 10^{5} / \mathrm{mL}$, when the cells reached $70 \%$ confluence (the time at which they would be exposed to UV), $12 \mathrm{~h}$ later the cells of the non-irradiated group would have reached a confluence in excess of $95 \%$. Therefore, we examined the effect of UV irradiation on keratinocyte-derived cytokines at $12 \mathrm{~h}$ after irradiation.

\section{Different effects of UVA and UVB on HaCaT cells}

UV has been shown without question to be the important inducer in the pathogenesis of photo-dermatitis. Previous studies regarding photo-dermatitis have focused primarily on the effects of UVB on keratinocyte-derived immunomodulating cytokines and adhesive mol- 
ecules (Grundmann et al., 2001). Although the biological activity of UVA is not as strong as that of UVB, it has a higher ratio in sunlight and deeper penetration. Accordingly, researchers have begun to consider the effects of UVA on photo-dermatitis (Krutmann, 2000). Our results indicated that the effects on keratinocyte-derived cytokines after UV exposure differed according to the dose of irradiation and the type of cytokines analyzed. For Th1s, the effect of $2.00 \mathrm{~J} / \mathrm{cm}^{2}$ UVA on HaCaT derived IL-10 was similar to that of UVB whereas the opposite effect was seen for $4.00 \mathrm{~J} / \mathrm{cm}^{2}$ UVA irradiation; the effect of UVA on HaCaT-derived IL-4 was similar to that of UVB. For Th2-type cytokines, the effects of UVA and UVB were similar. Current research results considering the photobiology of UVA are not entirely consistent, and a few are incompatible. Some scholars think that UVA might strengthen UVB. Serre et al. (1997) reported that covering the skin with sunscreen could adversely affect the contact hypersensitivity after UV exposure, and they therefore conjectured that UVA might cooperate with UVB. On the other hand, Kondo and Jimbow (1998) reported that keratinocytes could produce IL-12 after exposure to UVA, and suggested that IL-12 might inhibit the immunological suppression induced by exposure to UVB characterized by production of IL-10. Our results might be considered to reflect the former conclusions from some perspectives. Our analysis showed that different effects were produced in the course of keratinocyte-derived cytokine release, according to the different doses and the different composition of UV applied. Kondo and Jimbow (1998) also thought that the different UV composition primarily determined the balance of cytokine production between Th1 type and Th2 types.

\section{Effect of UV on keratinocyte-derived Th1 type cytokines}

Our previous research indicated that the infiltrating $\mathrm{T}$ cells in lesions from photodermatitis patients primarily expressed IL-12R and IFN- $\gamma$ R and that the secretion of IL-12 and IFN- $\gamma$ was higher than that of controls. Based on this result, to clarify the pathobiological change of keratinocytes after exposure to UV irradiation, we detected the effect of UV on HaCaT cell-derived Th1-type cytokines IL-12 and IFN- $\gamma$. Our results indicated that there was no or little expression of IL-12R and IFN- $\gamma \mathrm{R}$ in the HaCaT cells and the same result was seen in the supernatant $12 \mathrm{~h}$ after UVA and UVB irradiation. Although in our experiment we could not detect the secretion of IL-12 and IFN- $\gamma$, this did not exclude the possibility of other mechanisms preventing secretion of these cytokines, or that they might have been stored in the cells. In addition, the keratinocyte itself might secrete many kinds of cytokines in vivo; in turn they are also the target cells of different immunomodulators serving various functions toward influencing the cytokines and modulators secreted by themselves and other cells, allowing them to participate in the process of immunomodulation (Maas-Szabowski et al., 2003) Under our experimental conditions, we used $\mathrm{HaCaT}$ cells as the research objective, which could deviate the entire immune environment in vivo, and which therefore might cause it to not be directly applicable to understanding the biology behavior of keratinocytes (Tsuda et al., 2003).

\section{Effect of UV on keratinocyte-derived Th2 type cytokines}

On the basis of the previous conclusion, we detected the effect of UV on the HaCaT cell-derived Th1 type cytokines IL-4 and IL-10. Our results indicated that prior to irradiation, only weakly positive IL-10R expression and slight spontaneously secreted IL-10 could be detected, whereas, and with UVB irradiation, an up-regulation of both IL-10R and IL-10 could 
be detected $12 \mathrm{~h}$ after irradiation. A similar finding was observed in following lower dose UVA irradiation. Some studies have demonstrated that UV exposure can induce a systematic immunosuppression reaction, and that this reaction is related to keratinocyte-derived IL-10 (Rivas and Ullrich, 1992). It indicated that without stimulation, keratinocytes spontaneously secrete minute amounts of IL-10, and that the secretion is up-regulated after stimulation such as UV irradiation or allergic contact, and IL-10 negatively acts on the local immunoreaction. The observation that $I L-10$ gene knock-out mice are usually hypersensitive to allergens indicates that keratinocyte-derived IL-10 has some effect on sustaining the stabilization of the skin immune-environment (Rivas and Ullrich, 1992). IL-10 up-regulation can inhibit the occurrence of DTH and this inhibition is related to photo-carcinogenesis; this finding is consistent with our experimental results. Furthermore, our experiment indicates that keratinocytes might self-regulate their expression of IL-10R and IL-10. We suggest that the effects of the culture condition itself on keratinocytes should not be excluded when discussing the biology of keratinocytes in vitro. In addition, the lamp-housing of UV equipment and the doses of irradiation also impact the experimental results, which might be an important aspect to consider in the former, differing sets of results. IL-4 is another important Th2 cytokine. Our results suggested that the expression of IL-4R in HaCaT cells was up-regulated by cells exposed to higher dose of UVA and UVB; however, there was no change in IL-4 secretion into the supernatant and no IL-4R expression could be detected after lower dose UV exposure. It is generally thought that IL-4 is produced mainly from other epidermal or infiltrating inflammatory cells. However, keratinocytes express IL-4 mRNA and protein, and act as the target cells of IL-4 (Xiao et al., 2003). Our results are consistent with this conclusion.

In conclusion, Th1 and Th2 cytokines function oppositely in immunomodulation. In a pathological situation, keratinocytes up-regulate the local Th1 or Th2 in the skin by selectively secreting different functional cytokines. An imbalance of Th1/Th2 occurs in skin inflammatory disease. Our results suggest that $\mathrm{HaCaT}$ cells give priority to expression of $\mathrm{Th} 2$ cytokines at $12 \mathrm{~h}$ after UVB and $2.00 \mathrm{~J} / \mathrm{cm}^{2}$ UVA irradiation, up-regulate IL-4 and downregulate IL-10, and that there is no significant impact on Th1 cytokines after $4.00 \mathrm{~J} / \mathrm{cm}^{2} \mathrm{UVA}$ irradiation. This model seems to be the opposite of our research results obtained in vivo. Our former result (Li et al., 2007) suggests that the secretion of IL-12 and IFN- $\gamma$ in patient serum is higher than that in the normal control, and that there is no significant change in the secretion of IL-4 and IL-10. Our analysis of the result shows that there are all positive expression of IL-12R, IFN- $\gamma$ R, IL-10R, and IL-4R in HaCaT cells regardless of UV irradiation exposure, suggesting that keratinocytes play an important role in local immunity. While different UV compositions have different consequences for the various keratinocyte-derived cytokines, the different composition and doses of UV might have the net effect of balancing out the Th1/ Th2 cytokines. Our previous result was obtained in vivo and the research was done using infiltrating $\mathrm{T}$ lymphocytes in the dermis. This study used the HaCaT cell line and further discusses the pathogenesis of photo-dermatitis from the point of view of keratinocytes. These two phenomena, keratinocyte-mediated Th2 reaction following UV irradiation in vitro and lymphocyte-mediated Th1 reaction in vivo, might be spatially distinct, but might perhaps reflect a concerted result on the whole. We can assume that following UV irradiation, if priority was given to the keratinocyte-mediated Th2 reaction, it would induce immunosuppression which might lead to photo-carcinogenesis; on the other hand, if priority was given to the lymphocytemediated Th1 reaction, it would induces DTH which might lead to photo-dermatitis. These consequences are not contradictory. The present report about keratinocyte-derived cytokines 
is primarily based on results in vitro with the $\mathrm{HaCaT}$ cell line or on keratinocytes cultured in vitro. The conclusion drawn from in vitro studies might be different from that drawn from in vivo studies because of the difference of experimental conditions and the factors that affect each condition. Further study would be needed to determine whether this supposition is correct.

Our study investigated the relationship between cytokines derived from keratinocyte exposure to UV radiation and an imbalance of $\mathrm{T}$ helper cell subgroups. These results could provide experimental support for further studies on the pathogenesis of photo-dermatitis.

\section{Conflicts of interest}

The authors declare no conflict of interest.

\section{REFERENCES}

Aubin F (2003). Mechanisms involved in ultraviolet light-induced immunosuppression. Eur. J. Dermatol. 13: 515-523.

Chen H, Deng DQ, Zhou XH, Fan YY, et al. (2005). Imbalance of Tc cell in the pathogenesis of chronic actinic dermatitis. Chin. J. Dermatol. 38: 628-630.

Grundmann JU, Böckelmann R, Bonnekoh B and Gollnick HP (2001). UV erythema reducing capacity of mizolastine compared to acetylsalicylic acid or both combined in comparison to indomethacin. Photochem. Photobiol. 74: 587592.

Kondo S and Jimbow K (1998). Dose-dependent induction of IL-12 but not IL-10 from human keratinocytes after exposure to ultraviolet light A. J. Cell Physiol. 177: 493-498.

Krutmann J (2000). Ultraviolet A radiation-induced biological effects in human skin: relevance for photoaging and photodermatosis. J. Dermatol. Sci. 23: S22-S26.

Li HY, Deng DQ, Wang JH and Fan YJ (2007). Effects of UV on IL-12 and IFN- $\gamma$ derived from HaCaT cells. China J. Lepr. Skin Dis. 23: 1-3.

Maas-Szabowski N, Stärker A and Fusenig NE (2003). Epidermal tissue regeneration and stromal interaction in HaCaT cells is initiated by TGF-alpha. J. Cell Sci. 116: 2937-2948.

Mee JB, Alam Y and Groves RW (2000). Human keratinocytes constitutively produce but do not process interleukin- 18 . Br. J. Dermatol. 143: 330-336.

Mozzanica N, Cattaneo A, Trabattoni D, Finzi AF, et al. (1995). Production of type 1 and type 2 cytokines by peripheral blood mononuclear cells of psoriatic patients. Immunology 86: 422-426.

Rivas JM and Ullrich SE (1992). Systemic suppression of delayed-type hypersensitivity by supernatants from UVirradiated keratinocytes. An essential role for keratinocyte-derived IL-10. J. Immunol. 149: 3865-3871.

Schmitt DA, Walterscheid JP and Ullrich SE (2000). Reversal of ultraviolet radiation-induced immune suppression by recombinant interleukin-12: suppression of cytokine production. Immunology 101: 90-96.

Serre I, Cano JP, Picot MC, Meynadier J, et al. (1997). Immunosuppression induced by acute solar-simulated ultraviolet exposure with a sun protection factor of 15 and high UVA protection. J. Am. Acad. Dermatol. 37: 187-194.

Teraki Y, Hotta T and Shiohara T (2000). Increased circulating skin homing cutaneous lymphocyte associated antigen (CLA)+ type 2 cytokine producing cells, and decreased CLA+ type 1 cytokine producing cells in atopic dermatitis. Br. J. Dermatol. 143: 373-378.

Tsuda T, Tohyama M, Yamasaki K, Shirakata Y, et al. (2003). Lack of evidence for TARC/CCL17 production by normal human keratinocytes in vitro. J. Dermatol. Sci. 3: 37-42.

Xiao T, Kagami S, Saeki H, Sugaya M, et al. (2003). Both IL-4 and IL-13 inhibit the TNF-alpha and IFN-gamma enhanced MDC production in a human keratinocyte cell line, HaCaT cells. J. Dermatol. Sci. 31: 111-117. 